\title{
Supplementary file 1: Appendices
}

\section{Appendix A}

Table A1. Means, standard deviations, and bivariate correlations of the key variables

\begin{tabular}{|c|c|c|c|c|c|c|c|c|c|}
\hline & Variable & $M$ & $S D$ & 1 & 2 & 3 & 4 & 5 & 6 \\
\hline 1 & Societal discontent & 4.50 & 1.05 & - & $0.60 *$ & $0.53 *$ & $0.42 *$ & $-0.33 *$ & $0.72 *$ \\
\hline 2 & Intergroup threat & 4.66 & 1.48 & $0.60 *$ & - & $0.67 *$ & $0.41 *$ & $-0.64 *$ & $0.53 *$ \\
\hline 3 & Anti-refugee intentions & 2.64 & 1.42 & $0.53 *$ & $0.67 *$ & - & $0.57 *$ & $-0.46 *$ & $0.55 *$ \\
\hline 4 & $\begin{array}{l}\text { Refugee correcting } \\
\text { intentions }\end{array}$ & 3.38 & 2.04 & $0.42 *$ & $0.41 *$ & $0.57 *$ & - & $-0.13 *$ & $0.51 *$ \\
\hline 5 & Pro-refugee intentions & 3.49 & 1.39 & $-0.33 *$ & $-0.64 *$ & $-0.46 *$ & $-0.13 *$ & - & $-0.29 *$ \\
\hline 6 & $\begin{array}{l}\text { Anti-government } \\
\text { intentions }\end{array}$ & 3.54 & 1.76 & $0.72 *$ & $0.53 *$ & $0.55 *$ & $0.51 *$ & $-0.29 *$ & - \\
\hline
\end{tabular}

Note. ${ }^{*} p<.001$ 


\section{Appendix B}

\section{Explanation specification model 2}

Research has shown that violations of normality are common in latent interaction models, and alternative procedures have been suggested that are known to be more robust against these violations, especially when sample sizes are relatively high (e.g., Marsh et al., 2004; Moulder \& Algina, 2002).

We used a procedure in which the most reliable indicators of each latent predictor are centred and are multiplied once to yield a latent interaction variable (Wu et al., 2013). In contrast to other procedures (Kenny \& Judd, 1984), the variance or covariances with other variables were not constrained. The most reliable indicators of societal discontent were Lack of Trust, Negative Emotions, Negative Zeitgeist, and Societal Pessimism; the most reliable indicators for intergroup threat were item 5 (refugees as cultural enrichment), 1 (refugees damage norms and values), 2 (refugees are an economic benefit), and 3 (money is wasted on refugees). Thus, the indicators for the latent interaction variable were Pessimism multiplied by threat item 5, Distrust multiplied by threat item 1, Negative Societal Emotions multiplied by threat item 2, and Negative Zeitgeist multiplied by threat item 3 .

\section{References}

Kenny, D. A., \& Judd, C. M. (1984). Estimating the nonlinear and interactive effects of latent variables. Psychological Bulletin, 96(1), 201-210. https://doi.org/10.1037/0033-

\section{$\underline{2909.96 .1 .201}$}

Marsh, H. W., Wen, Z., \& Hau, K.-T. (2004). Structural Equation Models of Latent Interactions: Evaluation of Alternative Estimation Strategies and Indicator Construction. Psychological Methods, 9(3), 275-300. https://doi.org/10.1037/1082-989X.9.3.275 
Moulder, B. C., \& Algina, J. (2002). Comparison of Methods for Estimating and Testing Latent Variable Interactions. Structural Equation Modeling: A Multidisciplinary Journal, 9(1), 119. https://doi.org/10.1207/S15328007SEM0901_1 


\section{Appendix C}

Table C1. Measurement model of Model 2

\begin{tabular}{|c|c|c|c|c|c|c|}
\hline $\begin{array}{c}\text { Latent } \\
\text { construct }\end{array}$ & Observed variable & $\mathrm{B}$ & SE & $95 \% C I$ & beta & $\mathrm{p}$ \\
\hline \multirow{3}{*}{$\begin{array}{l}\text { Anti- } \\
\text { government }\end{array}$} & Firm, violent treatment & 0.93 & 0.02 & {$[0.90 ; 0.96]$} & 0.81 & $<.001$ \\
\hline & More aggression required & 0.95 & 0.02 & {$[0.92 ; 0.98]$} & 0.81 & $<.001$ \\
\hline & Overthrow the system & 1.11 & 0.01 & {$[1.09 ; 1.14]$} & 0.91 & $<.001$ \\
\hline \multirow{3}{*}{$\begin{array}{l}\text { Anti- } \\
\text { refugee }\end{array}$} & Avoid contact & 0.90 & 0.02 & {$[0.85 ; 0.94]$} & 0.70 & $<.001$ \\
\hline & Protect the neighborhood & 1.15 & 0.02 & {$[1.10 ; 1.19]$} & 0.83 & $<.001$ \\
\hline & Protest against refugees & 0.96 & 0.02 & {$[0.91 ; 1.00]$} & 0.80 & $<.001$ \\
\hline \multirow{3}{*}{ Pro-refugee } & Demonstrate for refugee-rights & 0.75 & 0.03 & {$[0.70 ; 0.81]$} & 0.61 & $<.001$ \\
\hline & Donate money or clothes & 1.15 & 0.03 & {$[1.09 ; 1.20]$} & 0.77 & $<.001$ \\
\hline & Teach or coach refugees & 1.10 & 0.03 & {$[1.05 ; 1.15]$} & 0.74 & $<.001$ \\
\hline \multirow{2}{*}{$\begin{array}{l}\text { Refugee- } \\
\text { corrective }\end{array}$} & Correct firmly & 1.03 & 0.01 & {$[1.01 ; 1.06]$} & 0.91 & $<.001$ \\
\hline & Correct with violence & 0.97 & 0.01 & {$[0.94 ; 0.99]$} & 0.91 & $<.001$ \\
\hline \multirow{5}{*}{$\begin{array}{l}\text { Intergroup } \\
\text { Threat }\end{array}$} & Causes housing problems & 0.75 & 0.02 & {$[0.71 ; 0.79]$} & 0.74 & $<.001$ \\
\hline & Cultural enrichment (recoded) & 0.91 & 0.02 & {$[0.87 ; 0.95]$} & 0.86 & $<.001$ \\
\hline & Damage to norms and values & 0.89 & 0.02 & {$[0.85 ; 0.93]$} & 0.82 & $<.001$ \\
\hline & Economic benefits (recoded) & 0.76 & 0.02 & {$[0.72 ; 0.80]$} & 0.79 & $<.001$ \\
\hline & Waste of money & 1.00 & 0.00 & {$[1.00 ; 1.00]$} & 0.88 & \\
\hline \multirow{4}{*}{$\begin{array}{l}\text { Societal } \\
\text { Discontent }\end{array}$} & lack of trust & 0.92 & 0.03 & {$[0.87 ; 0.97]$} & 0.81 & $<.001$ \\
\hline & negative emotions & 0.85 & 0.03 & {$[0.80 ; 0.91]$} & 0.72 & $<.001$ \\
\hline & negative zeitgeist & 0.67 & 0.03 & {$[0.61 ; 0.72]$} & 0.69 & $<.001$ \\
\hline & pessimism & 1.00 & 0.00 & {$[1.00 ; 1.00]$} & 0.84 & \\
\hline \multirow{4}{*}{ Interaction } & $\begin{array}{l}\text { lack of trust } x \\
\text { cultural enrichment }\end{array}$ & 0.82 & 0.07 & {$[0.69 ; 0.95]$} & 0.65 & $<.001$ \\
\hline & $\begin{array}{l}\text { negative emotions } \mathrm{x} \\
\text { damage to norms and values }\end{array}$ & 0.85 & 0.07 & {$[0.71 ; 0.98]$} & 0.61 & $<.001$ \\
\hline & $\begin{array}{l}\text { negative zeitgeist } \mathrm{x} \\
\text { economic benefits }\end{array}$ & 0.62 & 0.06 & {$[0.51 ; 0.73]$} & 0.63 & $<.001$ \\
\hline & $\begin{array}{l}\text { pessimism } \mathrm{x} \\
\text { waste of money }\end{array}$ & 1.00 & 0.00 & {$[1.00 ; 1.00]$} & 0.69 & \\
\hline
\end{tabular}

